\title{
MECHANISMS FOR RADIO CONTINUUM EMISSION OF LONG-PERIOD VARIABLE STARS
}

\author{
G. M. RUDNITSKIJ \\ Sternberg Astronomical Institute Moscow State University Moscow V-234, 119899 Russia
}

The radio continuum emission of long-period variables (LPVs) in the continuum is considered. For some LPVs (e.g., R Aql and V Hya) weak emission, observed at centimeter wavelengths, can be explained by thermal free-free radiation of the ionized gas behind the shock front in the stellar atmosphere. The ionized layer behind the shock remains optically thick at the wavelengths as short as $1 \mathrm{~cm}$; the brightness temperature found for $\mathrm{R} \mathrm{Aql}$ at $2 \mathrm{~cm}$ by Drake et al. (1987, AJ, 94, $1280), \mathrm{T}_{b}=18000 \mathrm{~K}$, is consistent with the expected temperature behind the shock.

For $\mathrm{R} \mathrm{Aql}$, at least two nonthermal radio continuum events (with flux densities up to $250 \mathrm{mJy}$ ) were recorded - in 1973 by Woodsworth and Hughes (1973, Nature, Phys. Sci., 246, 111) and in 1982 by Estalella et al. (1983, A\&Ap, 124, 309) I suggest as an explanation for these events synchrotron radiation by electrons accelerated at the shock front. The conditions for the shock acceleration in an LPV's atmosphere are discussed.

Amplification of radio continuum emission (both thermal and nonthermal) by masering molecules $\left(\mathrm{OH}, \mathrm{H}_{2} \mathrm{O}\right)$ in the circumstellar envelope may account also for the light-curve-correlated variability of circumstellar masers.

\section{HISTORY OF THE LIGHT CURVES AND MOLECULAR MASER EMISSION OF THE MIRAS U ORI AND R LEO}

\author{
I. L. ANDRONOV and L. S. KUDASHKINA \\ Odessa Astronomical Observatory Shevchenko Park Odessa, 270014 Ukraine \\ and \\ G. M. RUDNITSKIJ \\ Sternberg Astronomical Institute Moscow State University Moscow V-234, 119899 Russia
}

We have collected all the available data on light curves, $\mathrm{OH}, \mathrm{H}_{2} \mathrm{O}$ and $\mathrm{SiO}$ maser observations for a sample of Mira-type variables. We consider in detail the data on two stars, U Ori and $\mathrm{R}$ Leo. There is a net correlation between optical and radio line variations for all the three molecular species in these stars. More pronounced maser flares seem to follow brighter-than- average visual maxima of the stars. We discuss also the drastic changes in the type of the $\mathrm{OH}$ maser radio emission which happened in these stars some years ago. Implications for the mechanisms of maser pumping and the evolutionary status of these stars (probably undergoing the helium flash) are discussed. 